\title{
Développement humain, développement durable et " pays en développement » : comment articuler et mesurer les différentes dimensions?
}

\section{Bruno Boidin}

\section{(2) OpenEdition}

Journals

\section{Édition électronique}

URL : http://journals.openedition.org/developpementdurable/1700

DOI : 10.4000/developpementdurable.1700

ISSN : 1772-9971

Éditeur

Association DD\&T

\section{Référence électronique}

Bruno Boidin, « Développement humain, développement durable et « pays en développement »: comment articuler et mesurer les différentes dimensions ? », Développement durable et territoires [En ligne], Varia (2004-2010), mis en ligne le 01 février 2004, consulté le 19 avril 2019. URL : http:// journals.openedition.org/developpementdurable/1700; DOI : 10.4000/developpementdurable.1700

Ce document a été généré automatiquement le 19 avril 2019.

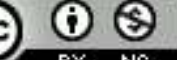

Développement Durable et Territoires est mis à disposition selon les termes de la licence Creative Commons Attribution - Pas d'Utilisation Commerciale 4.0 International. 


\title{
Développement humain, développement durable et « pays en développement »: comment articuler et mesurer les différentes dimensions?
}

\author{
Bruno Boidin
}

1 Si le concept et les objectifs de développement durable sont par nature universels, leur application ne peut être que différenciée en fonction des terrains étudiés. C'est sur ce principe que se fonde cet article, dont l'objectif est de questionner les dimensions (économique, environnementale, sociale) du développement durable au regard des contraintes des pays en développement, en insistant tout particulièrement sur la nécessité de considérer ces dimensions de façon intégrée plutôt que juxtaposée. Dans ce cadre, la dimension humaine, et en son sein la dimension sociale, tiendront une place centrale dans l'analyse.

2 Notre questionnement s'articule en trois axes, qui feront l'objet des parties successives. Le premier axe (section 1) cherche à préciser la place qu'occupent les différentes dimensions $\mathrm{du}$ développement durable au sein de l'analyse économique du développement, et en particulier les dimensions humaine et sociale. ${ }^{1}$ Ces précisions étant faites, la deuxième section s'intéresse aux relations particulières entre ces dimensions, dans un contexte de pays pauvres où le niveau initial de certaines composantes du développement humain (éducation, santé...) est particulièrement faible. Enfin la troisième section aborde le problème de la mesure des dimensions du développement durable dans une perspective internationale cherchant à comparer la situation des différents pays. 


\section{La place des dimensions humaine et sociale dans l'analyse économique du développement}

3 La recherche de la «soutenabilité » environnementale constitue un objectif souhaitable des sociétés, et justifie l'investissement des chercheurs et des décideurs dans ce domaine. Cependant les dimensions économique, humaine et sociale du développement durable restent souvent, dans le cas des économies en développement, des priorités. En effet la situation économique et sociale de ces pays implique de traiter prioritairement les questions de ressources globales disponibles (et de leur usage pour la production), et d'accès aux dotations de base (éducation, santé, habitat...). Ceci a amené les économistes et les institutions du développement à s'intéresser plus particulièrement à deux concepts :

- le développement humain, terme popularisé avec l'indice de développement humain (IDH) du PNUD,

- le développement social, venu ou revenu plus récemment dans les réflexions, et qui suppose que la sphère sociale tient une place centrale dans les conditions de développement durable.

Ces deux composantes participent à la dimension humaine du développement durable. Par dimension humaine du développement durable, on peut entendre la recherche d'un développement qui assure l'amélioration du bien-être humain (ou son maintien), ce dernier étant déterminé par des caractéristiques personnelles (éducation, santé, libertés individuelles...) ou collectives (cohésion sociale, niveau et répartition des richesses...). Le concept de développement humain recouvre donc plusieurs dimensions et constitue un champ de recherche encore récent et tâtonnant. En simplifiant, on y trouve à la fois :

- des travaux portant sur le développement humain au sens strict, en particulier le niveau de santé, d'éducation et de connaissances des individus ;

- et des recherches consacrées au développement social (voire socialement durable). Ce dernier concept nécessite quelques précisions, car cette dimension apparaît encore délaissée au sein des travaux sur le développement durable. Le développement socialement durable peut s'entendre comme un développement qui préserve (et si possible améliore) la cohésion sociale d'une société toute entière (et non pas seulement d'une partie de celle-ci), l'accessibilité des populations aux services sociaux et aux biens publics, la transmission des capacités aux générations futures. Si ces différentes améliorations constituent l'objectif implicite du développement, force est de constater que les politiques publiques peuvent également engendrer des conséquences néfastes, souvent non anticipées, dans le domaine du développement social. Les risques de « destruction du social » liés aux politiques publiques ne sont pas négligeables, mais de tels effets sont rarement examinés. Ceci amène certains auteurs (Dubois, Mahieu 2002) à proposer un principe de précaution sociale, obligeant les décideurs à évaluer ex ante les conséquences, dans le domaine social, des politiques menées.

Compte tenu des différentes dimensions à prendre ainsi en compte, l'économie du développement se trouve confrontée à deux difficultés majeures face à l'objectif de développement durable.

D'abord, les dimensions humaine et sociale du développement durable présentent encore des lacunes dans la définition des concepts, dans la spécification de leurs composantes, et dans la mesure de chacune d'entre elles. Ces difficultés sont certes également présentes 
dans les pays riches, mais elles touchent les pays pauvres avec plus d'ampleur, en particularité du fait des problèmes de recueil statistique et d'appréciation, tant qualitative que quantitative, de ces dimensions.

7 Ensuite, la recherche d'une prise en compte équilibrée des différentes dimensions du développement durable (environnementale, économique, humaine et sociale) est particulièrement délicate compte tenu de la situation économique et humaine des pays en développement. Dans un cadre de ressources limitées au niveau macroéconomique, la question posée à l'économiste du développement est de savoir s'il convient de procéder à des choix dans les priorités accordées aux différentes dimensions. Par exemple, la sortie de la pauvreté des populations les plus démunies (dimension humaine) pourrait être considérée comme prioritaire par rapport au développement économique ou à la non dégradation de l'environnement. Cependant il est difficile d'accepter une dégradation environnementale supplémentaire si elle rapproche le capital naturel d'un seuil de dégradation irréversible. De plus, même si de tels dilemmes venaient à être solutionnés, cette question est loin de se résumer à un simple arbitrage budgétaire et/ou politique entre différentes priorités (même si les plans d'ajustement structurel des années 1980-1990 ont semblé suivre cette voie restrictive, en favorisant les équilibres macroéconomiques au détriment du développement humain). En effet, les relations entre les différentes dimensions du développement durable sont complexes, car ces dimensions exercent une influence les unes sur les autres. On sait encore peu de choses, par exemple, sur les effets réciproques de l'environnement et du développement humain et social, même si l'on reconnaît que ces effets dépendent du niveau initial d'actif atteint par chacune de ces dimensions. On sait également peu de choses sur les relations entre le développement humain strict (santé, éducation...) et le développement social, même si l'on admet que ces deux composantes ne peuvent être étudiées indépendamment, car les actifs individuels ne sont évaluables que dans un contexte social donné.

8 En fin de compte, l'ambition des objectifs du développement durable se trouve confrontée, sur le terrain des pays en développement, à la difficulté d'améliorer simultanément et significativement le niveau de chaque dimension. Vaut-il alors mieux privilégier l'une ou l'autre dans un premier temps?

9 Les remarques précédentes suggèrent que cela n'est pas aussi simple, puisqu'en jouant sur une dimension on modifie potentiellement la situation au sein des dimensions voisines. Nous proposons, dans la partie suivante, de préciser ce problème des relations entre les dimensions du développement durable.

\section{La question des relations entre les dimensions et au sein des dimensions du développement durable}

10 La question des relations entre les différentes dimensions ou sous-dimensions du développement durable peut être abordée à partir d'une analyse en termes d'actifs. Les différentes dimensions, représentées par un stock initial, peuvent varier à la hausse ou à la baisse ${ }^{2}$. Bien entendu cette représentation n'a qu'une vocation analytique, dans les faits certaines composantes s'accordent mal avec une quantification.

11 Nous choisissons d'illustrer le problème à partir des liens entre la dimension humaine au sens strict (éducation, santé...) et la dimension sociale. La raison de ce choix tient au caractère particulièrement sensible de ces dimensions aux décisions de politique 
économique ou aux fluctuations économiques, compte tenu de la faiblesse des dotations humaines de base dans les pays en développement. Nous cherchons à souligner l'ampleur des problèmes d'irréversibilité dans un tel contexte.

\subsection{Les problèmes d'irréversibilité dans les économies en développement ${ }^{3}$}

12 Les différentes formes de capital (naturel, monétaire, humain, social) ne sont pas purement substituables, notamment dans le cas des actifs pour lesquels existent des seuils d'irréversibilité de la dépréciation. On peut examiner le cas des liens entre le capital social ${ }^{4}$ et le capital santé. Le choix de considérer le cas du capital santé (composante du capital humain) tient au rôle essentiel de ce dernier dans le développement humain et à sa faiblesse dans les économies en développement.

L'hypothèse de substitution possible d'un actif à l'autre ne peut être conservée que pour un certain niveau de capital. Les actifs sont substituables seulement si, pour chacun d'eux, le seuil d'irréversibilité n'a pas été atteint. ${ }^{5}$ Ainsi le capital social peut subir des dégradations irréversibles qui rendent impossible la compensation par d'autres formes d'actifs, monétaires ou non. De même, le capital santé peut conditionner la possibilité de recourir à d'autres formes d'actifs, comme l'a suggéré l'approche de Sen (1981) sur les dotations initiales (un stock trop faible de capital humain empêchera par exemple de recourir efficacement aux transferts ou aux aides matérielles communautaires). Prenons un exemple : dans le cas du SIDA en Afrique, on a constaté que la prise en charge familiale et communautaire (que l'on peut considérer comme un usage possible du capital social) permettait la survie, dans des conditions relativement satisfaisantes, des personnes touchées par la maladie. Cependant, cela n'est vrai que pour un état faiblement avancé de la maladie. Pour les cas avancés, la prise en charge communautaire est confrontée à l'insuffisance des ressources, à la remise en cause de l'équilibre communautaire et aux pressions émanant de l'entourage. Le capital social ne permet pas de compenser totalement la réduction du stock de santé.

14 Le problème est de définir les liens entre les seuils d'irréversibilité pour chaque type d'actif. Dans le cas du capital social et du capital santé, la combinaison de ces deux actifs, dans un but de maintien ou d'amélioration du bien-être, n'est possible qu'à partir d'un stock initial minimal de chacun d'entre eux. La difficulté est également de mesurer ces seuils croisés et ces effets de complémentarité, puisque les dotations en actifs sont différentes pour chaque individu, créant de multiples combinaisons possibles. On peut, par exemple, supposer que l'effet sur les populations d'une intervention jouant sur le capital social (politiques d'investissement-travail, projets de développement rural...) sera d'autant plus imprévisible que le capital humain de la population est initialement faible. Cela est vrai des populations d'Afrique subsaharienne, pour lesquelles les indices de développement humain sont parmi les plus bas. La faiblesse du capital humain étant partiellement compensée par le capital social, une remise en cause de ce dernier peut avoir des effets désastreux. C'est ainsi que certaines guerres civiles ont pu être interprétées comme les conséquences d'une pression trop forte sur le capital social, engendrant des clivages entre groupes sociaux homogènes qui jusque là vivaient en harmonie.

De là ne découle-t-il pas, pour les sociétés les plus pauvres, une priorité à donner au capital humain, notamment vers les besoins de base (santé, nutrition) ? L'insatisfaction de 
ces besoins empêche le maintien du capital social, ou fait peser la survie sur ce seul capital social (solidarité familiale, communautaire ou ethnique). Si le capital social joue un rôle essentiel de redistribution, en même temps il peut fragiliser l'individu dont la survie, ou l'accès aux dotations de base repose sur ce seul capital. En effet l'individu n'arbitre plus entre les diverses formes de capital. Le "portefeuille» d'actifs du plus pauvre se réduit donc aux transferts communautaires, qui peuvent être remis en cause en cas de forte pression économique (crise, plans d'ajustement structurel), politique (guerre...) ou environnementale. Il en découle la nécessité d'une combinaison plus équilibrée entre les différentes formes de capital, condition d'un développement réellement durable.

Les risques d'un capital social "surdimensionné " par rapport aux autres formes de capital ne remettent pas en cause ses effets potentiellement bénéfiques ${ }^{6}$. Cependant, ces risques ne doivent pas être négligés, en particulier lors de la mise en œuvre de politiques sectorielles favorables au "développement" du capital social dans les pays en développement. De telles politiques sont actuellement prônées par certaines institutions influentes (notamment la Banque mondiale), sous l'inspiration d'auteurs qui estiment que le manque ou la destruction de capital social constitue un frein au développement économique (on peut citer Collier et Gunning 1999, ou Stiglitz 1999).

Introduire le problème des seuils critiques entre les différentes formes de capital permettrait de préciser les liens entre capital social et capital humain, tout en dépassant l'idée répandue d'un capital social forcément trop faible. Comme il a été souligné, un capital social élevé associé à un capital humain faible peut augmenter les risques d'instabilité et de régression économique et sociale par le bais des conflits et déstructurations. Dans ce cas, la question n'est pas tant celle de l'importance du capital social, que celle de son usage (bienveillant ou malveillant) et celle de la répartition optimale entre le capital humain et le capital social (ou, plus généralement, entre les différentes formes de capital).

Cette question s'inscrit dans l'évolution récente des politiques de développement. Le passage des "plans d'ajustement » (années 80) à la « dimension sociale de l'ajustement » (Banque Mondiale, années 90) témoignait du rééquilibrage partiel entre les équilibres macro-économiques et le nécessaire développement humain. L'orientation ultérieure vers la "dimension institutionnelle du développement» (fin des années 90) répond à des préoccupations relatives au capital social (capital social issu cette fois des autorités et non de la communauté privée). Ces étapes des politiques du développement (de l'ajustement à la dimension sociale, puis institutionnelle) intègrent avec raison des dimensions jusque-là négligées ou oubliées, mais tiennent-elles compte du rapport entre capital humain et capital social, c'est-à-dire des seuils critiques permettant ou empêchant des substitutions entre ces deux actifs?

\subsection{Implications pour l'évaluation de la « durabilité » du développement.}

On le voit, l'articulation des dimensions du développement durable soulève des questions complexes dans le cas des pays en développement. Sans creuser plus avant ces questions dans le cadre de cet article, nous pouvons cependant en tirer une implication importante. Cette implication a trait à l'évaluation de la situation des pays en termes de durabilité. En effet la réflexion sur les dimensions de la durabilité (sections 1 et 2) ne peut être abordée 
indépendamment des questions d'évaluation du développement durable (section 3), puisque les problèmes d'articulation des différentes dimensions se retrouvent inévitablement dans les tentatives de mesure du développement durable.

Les développement précédents nous amènent à la conclusion suivante sur les indicateurs susceptibles de capter la situation relative d'un pays en termes de développement durable : il semble nécessaire d'utiliser conjointement des indicateurs synthétiques d'une part, et des indicateurs sectoriels d'autre part. En effet :

21 - Les indicateurs synthétiques (de type IDH) tentent de résumer en un chiffre la position des pays. Même s'ils ne fournissent qu'une vision globale, leur intérêt réside dans la possibilité qu'ils offrent de procéder à des comparaisons internationales de durabilité sur la base des "situations moyennes";

22 - Les indicateurs sectoriels (ou consacrés à une seule dimension du développement durable : environnementale, humaine...) permettent d'une part de prendre en compte les spécificités nationales (contexte environnemental, social, politique...), d'autre part de creuser la mesure de chaque dimension afin d'intégrer les possibles effets de seuil définis plus haut (dégradation irréversible de l'un des actifs, c'est-à-dire non compensable par la hausse d'un autre actif). Nous qualifierons ces mesures d'unidimensionnelles (si elles traitent par exemple la seule dimension humaine) ou de sectorielles (si elles mesurent une composante particulière d'une dimension, par exemple la santé).?

La troisième section aborde cette question de la mesure de la durabilité, en prenant en compte la nécessité d'une approche intégrant les différentes dimensions.

\section{La question de la mesure du développement durable}

24 Nous distinguerons les mesures unidimensionnelles (ou sectorielles) (3.1) des mesures synthétiques (3.2). Nous n'aborderons pas dans le détail les premières, mais insisterons sur leur intérêt face au problème de l'irréversibilité du stock d'actifs. Nous étudierons de façon plus approfondie les secondes, pour lesquelles nous proposerons des éléments d'évaluation.

\subsection{Les mesures sectorielles ou unidimensionnelles.}

Les mesures unidimensionnelles du développement durable s'intéressent à une dimension ou un secteur en particulier: par exemple les émissions polluantes pour l'environnement, l'espérance de vie et le taux d'alphabétisation pour le développement humain, les transferts entre vivants pour le capital social...

Pourquoi de tels indicateurs, bien que partiels face à la multidimensionnalité du développement durable, sont-ils néanmoins essentiels à toute mesure de la durabilité ? L'intérêt d'utiliser de tels indicateurs tient à la nécessité de dépasser la situation moyenne d'un pays pour entrer dans l'étude de chacune des dimensions. On peut illustrer cet argument en prenant l'exemple de l'IDH du PNUD.

L'IDH tente de synthétiser en une moyenne trois composantes du développement humain (monétaire, éducative et sanitaire). Or la critique souvent faite à l'IDH concerne le caractère réducteur d'une telle moyenne, qui ne reflète pas la diversité des situations dans chacune des dimensions de l'indicateur (santé, éducation, revenu). On peut, en outre, pousser plus loin cette critique en revenant sur une caractéristique beaucoup 
moins souvent évoquée et pourtant liée. Dans le calcul de l'IDH, il est nécessaire de considérer que les actifs du développement humain sont toujours substituables, de sorte que la faiblesse de l'un puisse être compensée par l'importance du ou des autres. Si cette caractéristique n'est pas clairement explicitée dans les rapports du PNUD, elle est néanmoins centrale et inhérente au calcul d'une moyenne.

A ce titre, l'IDH pose le problème de l'irréversibilité de la perte de certains actifs, qui n'est pas prise en compte dans le calcul. En effet les capacités individuelles dépendent de la possibilité d'accéder aux besoins essentiels, ce qui limite en partie les possibilités de substitution entre ces différents besoins. ${ }^{8}$ Il est donc nécessaire, au-delà de l'information fournie par l'indice synthétique moyen, de considérer la situation des pays au sein de chaque dimension.

Nous n'aborderons pas ici en détail les mesures unidimensionnelles, compte tenu de la spécificité de chacune d'entre elles et de la diversité des indicateurs possibles. Cependant certains de ces indicateurs seront évoqués ou abordés dans l'analyse des mesures synthétiques. En effet ces dernières intègrent différentes mesures unidimensionnelles.

\subsection{Les mesures synthétiques}

30 Après avoir précisé ce que l'on peut attendre des mesures synthétiques, et proposé des critères d'évaluation de ces dernières (a), nous confronterons de tels critères à une sélection d'indicateurs synthétiques (b).

\section{Que peut-on attendre des mesures synthétiques?}

31 Les mesures synthétiques du développement durable ne permettent pas de traiter le problème des seuils d'irréversibilité souligné plus haut (section 2). Elles ne poursuivent d'ailleurs pas expressément cet objectif, puisqu'elles sont d'abord destinées à une première comparaison internationale et, à terme, visent à influencer les décisions des régulateurs. ${ }^{9}$ C'est pourquoi nous les considérons comme complémentaires aux mesures unidimensionnelles ou sectorielles.

Que peut-on attendre des mesures synthétiques du développement durable ? ${ }^{10}$ Nous proposons d'établir une grille d'objectifs, qui servira également à fournir des critères d'évaluation de ces indicateurs. Quatre objectifs nous semblent pouvoir être proposés (pour chacun d'entre eux nous expliciterons les raisons de ce choix) :

- L'indicateur devrait intégrer les différentes dimensions pour lesquelles un consensus a émergé concernant les composantes du bien-être et du développement durable

- L'indicateur devrait permettre de procéder à des comparaisons entre pays et dans le temps

- l'indicateur devrait être un outil potentiel, direct ou indirect, de décisions publiques

- L'indicateur devrait permettre de prendre en compte les effets d'irréversibilité des différentes formes de capital.

Une remarque s'impose concernant ce dernier critère. Sa présence ici ne vise qu'à rappeler que les indicateurs synthétiques ne permettent pas de répondre à cet objectif, et qu'il convient de compléter ces derniers par des mesures sectorielles et nationales (cf. 3.1).Reprenons et explicitons les différents objectifs énoncés. 


\section{L'indicateur devrait intégrer les différentes dimensions pour lesquelles un consensus a émergé concernant les composantes du bien-être et du développement durable.} montre que ces indicateurs reposent généralement sur deux clés d'entrée complémentaires mais différentes :

- Soit l'on s'intéresse avant tout au bien-être, et le développement durable est alors une façon possible d'appréhender le bien-être au niveau d'une société. Dans ce premier cas, les critères et les dimensions de la durabilité constituent les dimensions du bien-être : le bien-être dépend d'un niveau de revenu suffisant (dimension économique), d'une instruction suffisante, d'un bon état de santé (dimension humaine), d'un environnement de qualité (dimension environnementale), d'une cohésion sociale forte (dimension sociale...).

- Soit l'on se situe d'emblée dans une préoccupation de développement durable, et les différentes dimensions citées ci-dessus sont étudiées sans que l'on recoure systématiquement à la notion de bien-être.

Dans le premier cas, on se trouve dans la «branche » de l'économie du bien-être, cette dernière pouvant recourir (et recourant assez massivement mais non exclusivement) aux dimensions du développement durable. Dans le second cas, on se situe dans l'économie du développement (si l'on est économiste) ou dans la thématique du développement durable (déclinée dans un certain nombre de disciplines). En réalité, ces deux angles d'attaque sont étroitement liés. Les économistes du "développement » et ceux du "bien-être » voient leur objet de recherche se croiser et se mêler étroitement sans toutefois se confondre. Ainsi l'on peut privilégier une approche centrée sur le développement humain (IDH), ce qui relève au départ de l'économie du développement, ou au contraire aborder la question sous l'angle du bien-être, ce qui nécessite de prendre en compte toutes ou partie des dimensions du développement durable.

Compte tenu de ces liens étroits entre bien-être et développement durable, nous parlerons ici d'indicateurs synthétiques de bien-être et de développement durable, afin de ne pas exclure certaines mesures qui, sans l'afficher explicitement, tentent en réalité de mesurer des dimensions de la durabilité. darité inter générationnelle (non remise en cause, voire recherche de l'élévation, du bien-être des générations futures), d'autre part la solidarité intra générationnelle (partage du bien-être ou des conditions de bien-être au sein d'une même génération). Ces deux principes se traduisent par l'énoncé normatif d'objectifs, qui composent les différentes dimensions de la soutenabilité ${ }^{12}$ : l'environnement (dimension centrale mais non exclusive de la solidarité inter générationnelle), la sphère économique, la dimension humaine et sociale, les inégalités et la pauvreté (ces dernières étant des dimensions centrales, mais également non exclusives, de la solidarité intra générationnelle).

On constate que les préoccupations d'ordre politique et civil (libertés politiques par exemple) et d'estime de soi (chère par exemple à Amartya Sen) ne sont pas directement présentes au sein de ces dimensions. Cela constitue une limite importante pour la mesure du bien-être car ces préoccupations constituent, à juste titre, un objet d'étude en

Développement durable et territoires , Varia (2004-2010) 
expansion et une dimension reconnue comme essentielle du bien-être. Cependant, même si doit être admise l'importance de ces composantes, la difficulté de leur mesure rend encore délicate leur prise en compte dans des indicateurs composites. Par ailleurs, ces dimensions du bien-être sont en partie déterminées par l'une ou l'autre des composantes du développement durable :

- ainsi l'estime de soi, bien qu'étant un sentiment subjectif, est en partie conditionnée par la satisfaction des besoins fondamentaux ;

- dans le même ordre d'idée, l'étude de Dasgupta et Weale (1992 p. 128) tend à montrer que, dans le cas des pays pauvres, les libertés politiques et civiles sont positivement corrélées avec le PIB, le taux de survie infantile et l'espérance de vie.

Pour ces différentes raisons, nous ne retiendrons pas directement ces dimensions civile, politique et d'estime de soi pour l'évaluation des indicateurs, cependant nous serons sensibles aux tentatives d'intégration de ces dimensions.

Il reste à savoir si toutes les dimensions ainsi évoquées peuvent être mesurées, et si un lien positif entre ces dimensions et le bien-être en général est avéré. Concernant cette seconde question, il semble que les analystes soient pour l'essentiel en accord sur l'effet bénéfique d'un revenu par habitant plus élevé (variable de dimension économique), d'un bon niveau de santé et d'instruction de base (variables de dimension humaine), d'un environnement non dégradé (dimension environnementale), enfin d'un partage des richesses socialement acceptable et d'une pauvreté faiblement étendue. A l'inverse, les travaux portant sur la dimension sociale du bien-être et/ou du développement durable sont contradictoires, en particulier pour ce qui touche au rôle du capital social (voir 2.1, ou Ballet et Guillon 2003). Compte tenu de ces incertitudes persistantes sur le rôle du capital social, et de l'absence actuelle de consensus, notre approche privilégiera la dimension humaine par rapport à la dimension sociale du bien-être. ${ }^{13}$

Compte tenu du premier objectif que l'on peut attribuer aux mesures synthétiques du développement durable, nous proposons d'évaluer les tentatives de mesure à l'aune du premier critère suivant :

43 Critère d'évaluation $\mathbf{n}^{\circ} \mathbf{1}$ : L'indicateur intègre-t-il les différentes dimensions pour lesquelles un consensus a émergé concernant les composantes du bien-être et du développement durable, à savoir :

- la dimension économique (niveau de revenu...)

- la dimension humaine au sens large (éducation, santé, et toutes les composantes du développement humain pouvant jouer sur l'estime de soi)

- la dimension environnementale

- les inégalités et la pauvreté.

\section{L'indicateur devrait permettre des comparaisons dans l'espace}

L'objectif poursuivi par les mesures effectuées à l'échelle mondiale, à savoir la réalisation de comparaisons internationales, contient en lui-même la nécessité de disposer d'indicateurs comparables dans l'espace. Cette comparabilité repose sur plusieurs conditions :

- les indicateurs utilisés doivent être disponibles ;

- les indicateurs utilisés doivent être pertinents compte tenu du niveau de développement de chaque pays. 
Ce deuxième point nécessite quelques précisions. La difficulté inhérente aux comparaisons internationales résulte de la difficulté à atteindre les deux objectifs qu'elles poursuivent généralement. Si les indicateurs sont spécifiques à un pays, ils peuvent capter assez finement les caractéristiques du pays étant donné son niveau de richesse, ses caractéristiques historiques, politiques et sociales. Si au contraire les indicateurs sont internationaux, ils constituent a priori des outils plus grossiers de mesure du bien-être. Comment alors refléter assez finement les situations nationales au regard des dimensions définies plus haut (critère $n^{\circ} 1$ ), tout en restant suffisamment simple et souple pour effectuer des comparaisons internationales? Nous verrons plus bas, dans l'examen des différents indicateurs sélectionnés, que tout dépend de l'objectif final: privilégier la finesse des indicateurs rend plus délicate la comparaison de tous les pays, alors que privilégier la simplicité fait perdre des informations précieuses pays par pays. Ce dilemme entre d'une part la comparabilité, d'autre part la précision de l'information contenue dans un indicateur international, nous suggère d'évaluer les différents indicateurs à l'aune de leur capacité à intégrer ces deux exigences, d'une part la prise en compte de particularités nationales ou locales, d'autre part le souci de comparabilité sur les grandes dimensions du développement durable et du bien-être. L'évaluation qui suivra plus bas vise également à estimer le degré de réalisme de ces exigences, compte tenu de l'état d'avancement actuel des initiatives.

Comment évaluer cette capacité à assurer à la fois la comparabilité et la prise en compte des spécificités ? Il nous semble que deux critères peuvent être privilégiés :

- premièrement, on peut supposer que c'est l'utilisation conjointe, dans chaque dimension, de données objectives (ou objectivées) et subjectives qui garantira de remplir ces deux conditions. Les premières sont utilisées pour faciliter la quantification des comparaisons (émission de CO2, espérance de vie, taux de pauvreté, de scolarisation, d'alphabétisation...). Les secondes, dont l'audience a augmenté ces dernières années, cherchent à tenir compte des perceptions propres aux populations, indépendamment de leur situation objective $;^{14}$

- deuxièmement, les indices synthétiques devraient être décomposables afin de retrouver et d'estimer la situation du pays dans chacune des dimensions. Ainsi les indicateurs synthétiques peuvent être composés d'indicateurs unidimensionnels ou sectoriels (cf. 3.1). Nous rejoignons ici les analyses portant sur les indicateurs internationaux de bien-être (Gadrey et Jany-Catrice 2003, Booysen 2002), qui semblent converger vers cette nécessité de privilégier des indicateurs à double volet : d'une part un indice composite, utile pour une première comparaison ou une vision générale de la situation des pays, d'autre part la possibilité d'étudier de façon désagrégée chaque dimension et chaque variable, afin d'affiner l'analyse par pays.

47 Critère d'évaluation $\mathbf{n}^{\circ} \mathbf{2}$ : Compte tenu de cet objectif de comparabilité dans l'espace, nous proposons donc de retenir cette comparabilité spatiale comme critère d'évaluation des indices synthétiques. L'évaluation peut, en particulier, porter sur :

- la disponibilité des données pour un nombre de pays significatif

- l'utilisation conjointe de variables « objectives » et « subjectives"

- la possibilité de désagréger les différentes dimensions et variables de l'indice composite

\section{L'indicateur devrait permettre des comparaisons dans le temps}

Les comparaisons dans le temps sont délicates, même dans le cas où les données sont disponibles. En effet, même lorsque un indicateur recouvre une période suffisamment 
longue pour permettre de telles comparaisons, il est fréquent que des évolutions dans le calcul de l'indicateur ou dans la nature de ses composantes engendrent des biais dans l'analyse inter temporelle. Or la comparabilité dans le temps semble essentielle à la pertinence d'une mesure du bien-être et du développement durable. Les progrès ou le déclin des indicateurs de bien-être dans le temps sont aussi importants que la situation de départ d'un pays. Parmi les arguments en faveur de cette proposition, on peut retenir le fait qu'une variation donnée d'une variable telle que le niveau d'éducation n'aura pas le même effet sur le bien-être, selon que la situation de départ est avantageuse ou au contraire difficile (il en est de même pour d'autres variables telles que la santé ou le revenu, ce qui a amené le PNUD à différencier l'effet d'accroissement de ce dernier selon le niveau de revenu initial).

Critère d'évaluation $\mathrm{n}^{\circ} 3$ : En conséquence la comparabilité dans le temps sera retenue parmi les critères d'évaluation des indicateurs internationaux de bien-être et de développement durable.

\section{L'indicateur devrait être un outil de décisions publiques}

Le dernier critère retenu est lié aux développements antérieurs relatifs à la comparabilité dans l'espace. Le dilemme soulevé plus haut (critère $n^{\circ} 2$ ) entre la simplicité de l'indice (permettant des comparaisons internationales) et sa finesse (permettant un examen plus fidèle de la situation de chaque pays) renforce la nécessité de savoir ce que l'on attend d'un indice à vocation internationale. Ici l'évaluation est plus délicate, car l'objectif des indicateurs n'est pas forcément affiché avec clarté : cherche-t-on à "bousculer » les indicateurs "dominants ", à enrichir les bases de données internationales, à tester la pertinence et la solidité des modèles sous-jacents?

51 Dans tous les cas, compte tenu de la demande sociale croissante pour des mesures du bien-être exploitables par les instances de régulation (pouvoirs publics, organismes internationaux...), un critère incontournable semble être la capacité à éclairer et aider la prise de décision de ces instances de régulation nationales ou internationales. ${ }^{15} \mathrm{Il}$ convient cependant de préciser que, dans le cas des indicateurs internationaux, cet usage d'outil pour la décision publique est le plus souvent indirect, voire encore ténu pour certaines mesures. En effet les difficultés techniques restent un obstacle majeur à l'élaboration d'indicateurs directement exploitables. Comme nous le verrons dans ce qui va suivre, ces indicateurs n'en sont qu'à leurs premiers pas.

Critère d'évaluation $n^{\circ} 4:$ Outil, direct ou indirect, de décisions publiques

53 Avant de procéder à l'évaluation, nous nous contentons de rappeler le dernier critère, dont la présence est justifiée plus haut (introduction du 3.2.1) :

Critère $\mathbf{n}^{\circ} 5:$ Prise en compte des effets d'irréversibilité des différentes formes de capital.

\section{b. Examen et évaluation de quelques tentatives de mesure}

Ce sont les indicateurs à vocation de comparaisons internationales qui feront l'objet de notre attention. Cela ne signifie pas que nous excluons a priori des initiatives intéressantes de mesures actuellement appliquées aux seuls territoires nationaux. En effet un certain nombre d'indicateurs nationaux pourraient être, ou ont été construits afin d'être utilisés à terme dans un cadre de comparaisons internationales. Cependant ces indicateurs restent pour le moment appliqués à un seul pays, ou à un groupe de pays 
considérés comme homogènes selon des caractéristiques données (par exemple un revenu par habitant proche). ${ }^{16}$

Si la liste des tentatives de mesure du bien-être et du développement durable à l'échelle mondiale s'est allongée depuis les années 1990, force est de constater que les difficultés rencontrées lors de ces initiatives rendent ces expériences encore tâtonnantes, voire confidentielles. Seul l'IDH du PNUD est devenu, sur une seule dimension (le "développement humain»), une référence internationale, quoique contestée par beaucoup d'analystes. Cela ne réduit cependant pas l'intérêt des autres initiatives. En effet ces dernières s'inscrivent dans un objectif d'amélioration des indicateurs, et d'aide à l'amélioration des connaissances sur les inégalités de bien-être entre les pays.

Nous présenterons ici, dans leurs grands principes, six d'entre elles. Ces six tentatives « universalistes » de mesure du bien-être et du développement durable constituent, à notre connaissance, les initiatives les plus avancées et les plus significatives. ${ }^{17}$ Nous choisissons de regrouper ces indicateurs en trois ensembles:

- - l'IDH, dont le caractère incontournable, tant comme indicateur précurseur que comme source de débats et donc d'avancées dans les recherches en cours, justifie d'être examiné isolément des autres mesures $;^{18}$

- - trois indicateurs alternatifs à l'IDH, mais présentant le même point commun de proposer une pondération arbitraire des différentes dimensions retenues : l'Index of Human Progress, le Quality of Life Index, enfin le Dashboard index. Ces trois indicateurs présentent bien entendu des différences, qui sont pour certaines d'entre elles importantes, par exemple dans les dimensions privilégiées. Cependant le choix d'une pondération arbitraire des dimensions nous semble un point de convergence suffisamment important pour identifier ensemble ces trois initiatives. En effet, au sein des indicateurs existants, le mode de pondération fait l'objet de débats intenses. On peut grossièrement distinguer les auteurs et analystes qui assument un choix, souvent subjectif, dans le poids accordé à chacune des dimensions du bien-être présentes dans l'indicateur ; et ceux qui, à l'inverse, estiment qu'il convient de fonder ce poids sur une méthode plus objective. Ce débat n'est pas clos, et il ne constitue pas l'objet principal du présent travail. Cependant cette question ne peut être ignorée ;

- - enfin deux indicateurs qui, à l'inverse des précédents, reposent sur une tentative de pondération « non arbitraire » des différentes dimensions : nous examinerons l'Index of Social Progress et l'approche par les fonctions distances ${ }^{19}$.

Le tableau 1 ci-dessous présente quelques caractéristiques des indicateurs évoqués. 
Tableau 1 : Indicateurs « universalistes » de bien-être et de développement

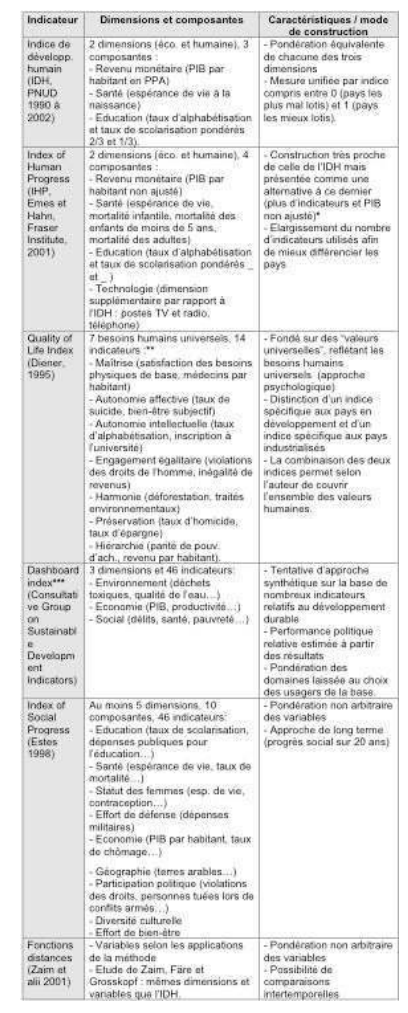

*Les auteurs reprochent à l'IDH le fait d'utiliser un PIB ajusté par des données logarithmiques. Ce choix provient de l'hypothèse que, au-delà d'un certain niveau, la contribution supplémentaire du PIB au bien-être devient faible. Emes et Hahn contestent ce choix, arguant le fait que celui-ci distord les résultats, et en particulier réduit l'impact du PIB dans l'indicateur global.

** Les variables indiquées entre parenthèses sont différenciées selon le niveau de développement : la première est celle retenue pour les pays en développement, la seconde celle retenue pour les pays industrialisés.

***http://esl.jrc.it/envind/dashbrds.htm

Nous tentons de présenter dans le tableau 2 ( $c f$. infra) la position des différents indicateurs vis-à-vis des critères d'évaluation.

Quel bilan peut-on tirer de ce tableau concernant la pertinence et l'utilité des indicateurs de bien-être et de développement durable? Pour apporter quelques réponses à cette question, nous reprenons chacun des critères prédéfinis.

64 Concernant le premier critère (intégration des dimensions du bien-être et du développement durable), la plupart des indicateurs cherchent à enrichir la mesure, soit en approfondissant la connaissance des dimensions déjà abordées dans l'IDH (dimensions économique et humaine), soit en investissant d'autres dimensions (dimension environnementale, dimension politique et des libertés humaines, estime de soi vue à travers le bien-être subjectif...).

Cette recherche d'un enrichissement de la mesure vise également à affiner les comparaisons internationales tout en rendant possible un examen plus détaillé des situations nationales. Mais elle se heurte alors à un obstacle qu'aucune mesure n'est 
encore parvenue à franchir. La complexification rend plus difficile la comparabilité compte tenu :

- de la difficulté à recueillir des données fiables, présentes dans chaque pays, et sur une longue période ;

- de la difficulté à internationaliser (homogénéiser entre les pays) les mesures subjectives du bien-être.

Ceci nous amène au deuxième critère, celui de la comparabilité dans l'espace. Les remarques précédentes nous montrent que l'effort actuel de recueil et de compilation de données par les organismes internationaux, centres de recherche et pouvoirs publics ne portera ses fruits qu'à long terme. En attendant de disposer d'un ensemble de données homogènes, l'utilisation d'indicateurs composites simples combinée à des approches sectorielles (environnement, éducation, santé, inégalités, libertés...) semble constituer la voie la plus souhaitable.

Le troisième critère (comparabilité dans le temps) permet de distinguer entre les indicateurs « photographiques » (mesures à un moment donné, telles que le Dashboard) et ceux qui à l'inverse sont construits dans un objectif de comparaisons inter temporelles (ISP de Estes). Entre ces deux cas de figure, certains indicateurs font l'objet de comparaisons dans le temps sans être initialement construits à cet effet (IDH), ce qui peut poser des difficultés méthodologiques que d'autres approches se proposent de solutionner (fonctions distance). La question de la comparabilité intertemporelle reste donc un problème crucial des mesures du bien-être et du développement durable.

Tableau 2 : Application des critères d'évaluation aux indicateurs universalistes

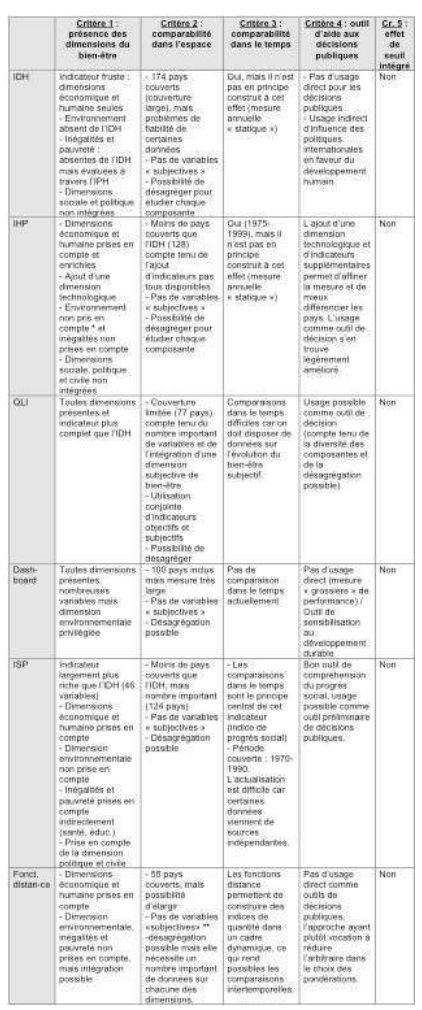

* L'absence de l'environnement est justifiée par les auteurs à partir de l'idée que, contrairement à l'affirmation du PNUD, il existerait une corrélation positive entre le PIB 
et la qualité de l'environnement, à partir d'un certain niveau de revenu (Emes et Hahn p. 13).

** Des approches similaires appliquées à un seul pays ont intégré une mesure subjective du bien-être, tout en soulignant les difficultés rencontrées (cf. notamment Pavone 1998, p.11). Cependant, même si ces difficultés peuvent être surmontées à cette échelle, la mesure du bien-être subjectif est beaucoup plus délicate à l'échelle internationale compte tenu des problèmes de recueil statistique de ce type de données.

L'utilisation des indicateurs comme outils de décisions publiques (quatrième critère) demeure à ce jour difficile, même si certaines initiatives (IHP, ISP) cherchent à dépasser les limites de l'IDH à cet égard. Cependant cette observation assez pessimiste sur l'impact politique peut être nuancée pour deux raisons :

- - d'abord, c'est au niveau des organisations internationales que l'influence de ces mesures peut être évaluée. Les rapports et initiatives de ces organisations depuis les années 1990 montrent une sensibilité variable mais certaine aux mesures de développement et de bienêtre qui intègrent les dimensions non monétaires (voir par exemple OCDE 2001) ;

- - ensuite, l'influence des mesures enrichies du développement se traduit d'abord dans les réflexions et les idées, avant de percer dans les stratégies et programmes politiques. Ce cheminement prend du temps et l'IDH ne constitue qu'une première étape de ce mouvement.

71 Enfin le cinquième critère (prise en compte des seuils d'irréversibilité) n'est pas pris en compte par les mesures synthétiques, ce qui justifie en retour l'utilisation conjointe des indicateurs sectoriels ou unidimensionnels. Ce problème est lié au deuxième critère, celui des comparaisons dans l'espace. Face au problème d'irréversibilité et de relations ambiguës entre les actifs, les comparaisons dans l'espace demeurent dans tous les cas un outil limité puisqu'elles ne permettent pas de cerner les risques d'insoutenabilité au niveau d'un pays.

\section{Conclusion}

Trois axes de réflexion ont été abordés dans cet article : la question des dimensions du développement durable privilégiées en économie du développement, le problème des relations entre ces dimensions, enfin les difficultés et les perspectives de mesure de ces dimensions dans une optique de comparaisons internationales. Les conclusions très provisoires auxquelles nous sommes parvenus peuvent maintenant être rappelées.

73 En premier lieu, l'ordre des priorités du développement durable au sein des pays à faible revenu se différencie de celui souvent privilégié dans les pays riches. Les dimensions humaine et économique constituent des enjeux cruciaux qui occultent encore en partie les préoccupations environnementales. Cette différence de priorité ne préjuge pas des effets négatifs des dégradations environnementales dans les pays pauvres, elle constitue simplement un fait au sein des stratégies prônées et des programmes. En outre, si la dimension sociale semble gagner progressivement du terrain au sein des pays industrialisés, sa prise en compte y reste très différente des modalités d'application dans les pays en développement, compte tenu des contextes et des contraintes spécifiques de ces derniers.

74 En deuxième lieu, les différentes dimensions du développement durable tendent actuellement à être considérées comme additives (on suppose que leurs effets sont 
mutuellement bénéfiques), au sein notamment des organismes internationaux. De nombreux acteurs du développement estiment que l'intervention sur l'une des dimensions n'aura pas d'effet pervers sur d'autres dimensions. Nous avons tenté d'insister, au contraire, sur les risques liés à la non prise en compte de possibles effets négatifs. Cette voie pourrait être creusée dans le cadre de travaux tentant de mieux appréhender les effets de seuil dans chaque dimension du développement durable.

En troisième lieu, la question du sens des relations entre les dimensions du développement durable nous a amené à aborder le problème de la mesure de ces dimensions. Nous avons souligné la nécessité de combiner des mesures unidimensionnelles d'un côté, et des mesures synthétiques de l'autre. L'intérêt des premières tient au fait qu'elles offrent une appréhension plus fine des situations nationales, permettant d'une part de mieux cerner les difficultés dans l'atteinte des objectifs de durabilité, d'autre part d'estimer ex ante les effets de seuil propres à chaque dimension. Concernant les mesures synthétiques, malgré leurs limites, nous défendons leur place au sein des indicateurs de bien-être et de développement durable. Certes, elles restent marginales et font encore l'objet de vives critiques, voire de condescendance au sein des économistes. Certains verront dans ces initiatives des tentatives vaines et trop ambitieuses, d'autres feront remarquer que la corrélation entre le niveau de revenu et les autres indicateurs rend inutile la recherche de mesures qualitatives du bien-être. Sans nier les difficultés et parfois les incohérences ou les risques liés à de telles approches, nous n'aboutissons pas à de telles conclusions. A condition de spécifier leurs objectifs, de clarifier leur méthodologie et de préciser leurs limites internes, les initiatives examinées nous semblent importantes pour deux raisons essentielles. D'une part, elles répondent à la nécessité d'offrir aux instances internationales de régulation des outils qui dépassent les mesures strictement monétarisées. D'autre part, elles fournissent une première information, certes imparfaite et grossière, mais utile à la sensibilisation et à la prise de décision des acteurs économiques et politiques dans le domaine du développement social et humain.

\section{BIBLIOGRAPHIE}

BALLET J., Guillon F.R. (ed.) (2003), Regards croisés sur le capital social, l'Harmattan.

BOOYSEN F. (2002) “An overview and evaluation of composite indices of development", Social Indicators Research, $59: 115-151$.

CMED (1989), Notre avenir à tous, Editions du Fleuve, Montréal.

Collier P., Willem GunNing J. (1999), “Explaining African Economic Performance”, Journal of Economic Literature, vol. XXXVII (March), pp. 64-111.

DAsGupta P., WEAlE M. (1992) “On measuring the quality of life”, World Development, vol. 20, n 11 : 119-132 
DASGUPTA P. (1990) “Well-being and the extent of its realization in poor countries", The Economic Journal, $\mathrm{n}^{\circ} 100: 400$.

DIENER E. (1995), "A value based index for measuring national quality of life", Social indicators research, $36: 107-127$

DUBoIS J.L., MAHIEU F.R. (2002), « La dimension sociale du développement durable : réduction de la pauvreté ou durabilité sociale? ", in Le développement durable?, Paris, IRD, 2002.

EMES J., HAHN T. (2001), Measuring development. An index of human progress. Fraser Institute occasional paper, $\mathrm{n}^{\circ} 36$.

ESTES R.J. (1998), “Trends in social development, 1970-1995 : challenges for a new century”, Journal of developing societies, 14(1) : 11-39

GADREY J., JANY-CATRICE F. (2003), Les indicateurs de richesse et de développement. Un bilan international en vue d'une initiative française. Rapport de recherche pour la DARES, mars.

HARTWICK J. (1978), “ Substitution among exhaustible resources and intergenerational equity ”, Review of Economic Studies, vol. 45, $\mathrm{n}^{\circ} 2$.

KAKWANI N. (1993), "Performance in living standards, an international comparison", Journal of development economics, vol. 41, $\mathrm{n}^{\circ} 2: 307-336$

MAHIEU F.-R., BOIDIN B. (2002), “Capital social, capital humain et principe de précaution”, in EADIGEMDEV, L'Europe et le Sud à l'aube du XXIème siècle : enjeux et renouvellement de la coopération, cédérom, Karthala.

MAZUMDAR K. (1999), "Measuring the well-being of the developing countries : achievement and improvement indices", Social Indicators Research ; 47 : 1-60

MIRINGOFF M., MIRINGOFF L. (1999), The social health of the nation, how America is really doing ? Oxford University Press

NORDHAUS W., TOBIN J. (1971), “Is growth obsolete ?”, Cowles Foundation Discussion papers n 319

OCDE (2001), Du bien-être des nations. Le rôle du capital humain et social. Paris.

OSBERG L., SHARPE A. (2002), “An index of economic well-being for selected OECD countries”, Review of Income and Wealth, vol. 48, $\mathrm{n}^{\circ}$ 3, 291-916

PAVONE A. (1998) "Measuring inequality in Italy. A non traditional approach", Paper presented to the Third Biennal Georgia productivity workshop, Athens, Georgia, october 1998.

PeARCE D., ATKInson G.D. (1993), “ Capital theory and the measurement of sustainable development : an indicator of “ weak" sustainability ", Ecological Economics, vol. 8, n 2 PEARCE D., TURNER K. (1990), Economics of natural resources and the environment, Harvester Weatsheaf, Londres, 1990.

PNUD (1990 à 2002) Rapport annuel sur le développement humain, divers éditeurs.

PUTNAM R. (2000), Bowling alone, the collapse and revival of american community, Simon and Schuster, New York

SEN A. (1993) “Positional objectivity", Philosophy and Public Affairs, vol. 22.

SEN A. (1981), Poverty and famines. An essay on entitlements and deprivation. Oxford, Clarendon Press. 
SHARPE A. (1999), “A survey of indicators of economic and social well being”, Background paper prepared for Canadian Policy Research Network, july 22.

STIGLITZ J., (1999), “Quis custodiet ipsos custodies”, Annual bank conference on development economics - Europe, June.

TURNBULL, J. (1972), Les Iks, Survivre par la cruauté, Paris, Plon/ terre humaine.

ZAIM O., FARE R., GROSSKOPF S. (2001) "An economic approach to achievement and improvement indexes", Social Indicators Research, $56: 91-118$

\section{NOTES}

1. Rappelons que les concepts de développement et de développement durable ne sont pas unanimement partagés, que ce soit au sein des économistes ou dans les autres disciplines des sciences humaines et sociales. Cependant notre article n'a pas pour cadre d'étude les discussions sur la pertinence de ces concepts. Nous considérons en effet que les insuffisances et les biais de la notion de développement durable, même s'ils sont indéniables, ne remettent pas en cause la pertinence d'ensemble du concept et sa capacité à susciter des avancées dans l'analyse et la connaissance.

2. C'est la démarche retenue dans les travaux qui étudient l'évolution des formes de capital dans le temps. Cette démarche constitue un point commun entre des approches très différentes, voir par exemple le modèle de Hartwick (1978) revu par Pearce et Atkinson (1993), pour ne citer que ces auteurs.

3. Ce paragraphe reprend et aménage certains passages d'un article précédent rédigé en collaboration avec F.R Mahieu (Mahieu et Boidin 2002).

4. «Le capital social est du revenu social escompté ou capitalisé pour une période et un taux d'intérêt donnés. Il est le fruit d'un investissement social qui crée un stock de droits et obligations selon Gluckman (1965), et éventuellement du revenu [...]. Le capital social est donc une capitalisation de droits interpersonnels (vis à vis d'autres personnes). Il diffère du capital humain (Schultz, 1960 ; Becker, 1964) qui concerne un stock personnel d'éducation et de santé, lequel peut être consommé ou augmenté. Les droits peuvent être considérés bruts ou nets des obligations qui sont dues. Ils peuvent donner lieu à des transferts de biens, de monnaie, d'éducation, d'information, de convictions religieuses, politiques, de proximité, d'affection. Il existe des droits et des transferts matériels et immatériels. La mesure du capital social par les réseaux de sociabilité pose de sérieux problèmes théoriques et pratiques; il est plus facile de mesurer certains flux : transferts par exemple ou revenus de pluriactivité » (extrait de Mahieu et Boidin 2002)

Références : Gluckman M. (1965), Politics and ritual in tribal society, Oxford, Blackwell; Schultz T.W. (1960), "The formation of human capital by education ", Journal of Political Economy, 68 (december); Becker G. (1964), Human capital: a theoretical and empirical analysis, New York, Columbia University Press.

5. Des auteurs tels que Pearce et Turner (1990) avaient énoncé ce principe pour le capital naturel, dans leur modèle de «soutenabilité forte ».

6. Le capital social peut donc être bénéfique, mais ne l'est pas systématiquement.

7. Cependant la mesure de certaines composantes pose problème, par exemple le capital social.

8. La particularité de la santé sous cet angle est d'être l'un des actifs les plus sensibles à la propriété d'irréversibilité. A titre de comparaison l'éducation peut être considérée comme inaltérable une fois qu'elle est acquise. On ne peut certes nier la dépréciation des connaissances 
dans le temps (facteurs physiologiques et techniques), mais cette dégradation ne tend à concerner que des savoirs non fondamentaux à l'inverse de l'écriture, du calcul ou de la lecture.

9. C'est pour cette raison qu'elles constituent un enjeu important au sein des organismes internationaux (Banque Mondiale, PNUD, OMS...) qui interviennent dans le domaine du développement.

10. Nous parlerons également de mesures universalistes. Par mesure universaliste, nous entendons une approche qui considère comme possible de proposer une évaluation à l'échelle internationale sur la base de critères communs. Ce type de mesure existe mais fait l'objet de critiques, au même titre que celles formulées à l'encontre des mesures monétaires du bien-être.

11. Nous situons notre analyse dans un cadre mondial, considérant que l'étude et l'intervention sur les pays en développement ne peut être menée qu'en considérant les rapports existants entre l'ensemble des pays, donc à l'échelle mondiale.

12. Par énoncé normatif d'objectifs nous entendons l'ensemble des textes et déclarations à visée normative, publiés notamment à partir du rapport «Notre avenir à tous » (CMED 1989), à l'initiative des Etats, des organisations internationales, des ONG.

13. Cela ne signifie pas que nous réduisions la dimension sociale à l'étendue du capital social. Cependant ce dernier en constitue une composante essentielle.

14. Il est probable que le succès des travaux de Amartya Sen ne soit pas étranger à ce phénomène. En particulier, le concept d'objectivité située proposé par l'auteur (Sen 1993) reflète bien la nécessité de considérer tout autant les conditions objectivement universelles du bien-être (le minimum dont chacun doit disposer) que l'utilisation située des ressources par chacun.

15. Bien entendu les décisions prises par les instances de régulation mériteraient un examen approfondi et prenant en compte les valeurs et les objectifs propres à chacune d'entre elles. Même si cet examen ne constitue pas l'objet de cet article, on peut citer les critères proposés par Gadrey et Jany-Catrice (2003, pp 151-152) pour évaluer les indicateurs en termes de décisions politiques. Les auteurs retiennent quatre critères sous forme de questions : une valorisation du résultat final est-elle proposée, et si oui laquelle ? L'indicateur a-t-il un objectif clairement défini de politique économique? Quels sont ses usages possibles (contrôle, rapport social, plaidoyer pour le débat public...) ? Quelle est sa légitimité, sa diffusion médiatique, son influence dans le débat public? Appliquée aux indicateurs utilisés par les acteurs internationaux influents et parfois concurrents (Banque mondiale, FMI, PNUD, OMC, ONG...), cette grille donnerait un éclairage particulièrement intéressant sur la façon dont de tels indicateurs sont utilisés, voire sur les différences d'usages d'un même indicateur.

16. Des typologies récentes d'indicateurs de bien-être et/ ou de développement ont été réalisées (Gadrey et Jany-Catrice 2003, Booysen 2002, Sharpe 1999). Leur objet premier n'étant pas l'application à l'échelle mondiale, elles reposent sur d'autres critères que celui que nous retenons, et détaillent certains indicateurs que nous ne ferons qu'évoquer, tout en abordant succinctement certaines initiatives qui sont centrales dans le présent article.

17. Nous n'abordons pas les mesures en termes de PIB corrigé (voir par exemple la mesure du Genuine savings de la Banque mondiale), qui cherchent à compléter le PIB par l'ajout ou le retrait de certaines dimensions de bien-être. Trois raisons expliquent cette absence. D'abord notre objectif ici n'est pas l'exhaustivité mais plutôt la recherche des limites et des pistes à creuser au regard des différentes initiatives. Ensuite le succès de l'IDH nous a orienté vers l'analyse des mesures synthétiques non monétaires plutôt que monétaires. Enfin, et c'est peut-être la raison la plus arbitraire, nous sommes plus réservés vis-à-vis des mesures monétarisées du bien-être même si elles constituent des tentatives intéressantes d'enrichissement du PIB.

18. L'IDH, tout en étant reconnu comme une avancée importante, est également contesté par certains analystes et pays en développement, qui estiment en particulier que les dimensions retenues et leur poids respectif témoignent de la subjectivité d'un tel indicateur. 
19. Une remarque s'impose concernant cette caractéristique des méthodes évoquées. Si le caractère non arbitraire de la pondération est bien établi sur le plan technique, il convient cependant de relativiser cette particularité. En effet, les méthodes en termes de fonctions distances reposent sur la mesure d'une efficacité technique. Ce critère d'efficacité technique permet de positionner les différents pays les uns par rapport aux autres. Bien entendu une telle approche se fonde sur des présupposés théoriques (notamment le raisonnement en termes d'input-output) qui ne sont pas exempts de choix arbitraires. En d'autres termes, la rigueur méthodologique et technique de ces approches ne doit pas faire oublier qu'elles reposent sur des modèles économiques sous-jacents qui font débat au sein des économistes.

\section{RÉSUMÉS}

Cet article cherche à préciser la place qu'occupent les différentes dimensions du développement durable au sein de l'analyse économique du développement, et en particulier les dimensions humaine et sociale. Ceci nous amène à considérer les relations complexes entre les différents actifs (santé, éducation, capital social...), marquées par des effets d'irréversibilité qui peuvent remettre en cause la durabilité. Se pose alors la question de la mesure du développement durable prenant en compte ces articulations entre les dimensions. Une grille d'évaluation des mesures synthétiques du développement durable est proposée et appliquée à une sélection d'indicateurs.

This article seeks to specify the place of various dimensions of sustainable development within development economics, in particular human and social dimensions. This leads us to consider the complex relations between the various assets (health, education, social capital...), characterized by irreversibility effects which can jeopardize sustainability. It is therefore necessary to find a measure of sustainable development, taking into account these interactions between dimensions. We propose criteria to evaluate synthetic measurements of sustainable development, and apply them to selected indicators.

\section{INDEX}

Mots-clés : développement humain, pays en développement, effets de seuil, indicateurs de bienêtre et de développement

Keywords : human development, developing countries, threshold effects, indicators of well being and development

\section{AUTEUR}

\section{BRUNO BOIDIN}

Bruno Boidin est maître de conférences en économie, membre du CLERSE, Université Lille 1. Il est par ailleurs membre du comité de lecture de la revue DDT. 